\title{
Le phénomène du masquage dans le signalement des événements « les plus graves » : biais influant sur les estimations des taux d'incidence des blessures sportives chez les enfants canadiens
}

\author{
A. Gupta, B. Sc. (1); C. M. Davison, Ph. D. (1,2); M. A. Mclsaac, Ph. D. (1,3)
}

Cet article a fait l'objet d'une évaluation par les pairs.

Diffuser cet article sur Twitter

\section{Résumé}

Introduction : Les enquêtes sur la cueillette de données sur les blessures ciblent souvent "l'événement le plus grave » dans le but de limiter les erreurs de mémoire et de réduire la longueur des questionnaires. Cela risque cependant de masquer des blessures moins graves et d'entraîner un biais dans les estimations des taux d'incidence de certaines sous-catégories de traumatismes.

Méthodologie : Nous avons utilisé des données tirées de l’Enquête sur les comportements liés à la santé des enfants d'âge scolaire (ECSEAS) et du Système canadien hospitalier d'information et de recherche en prévention des traumatismes (SCHIRPT) pour comparer les estimations des taux d'incidence des blessures sportives chez les enfants canadiens.

Résultats : D’après les données de l'ECSEAS, 6,7 \% des enfants déclarent avoir subi une blessure sportive ayant nécessité une consultation à un service des urgences. N'ont cependant été recueillies que les données sur la blessure « la plus grave » subie par l'enfant, ce qui fait qu'un enfant ayant subi de multiples blessures justifiant une consultation à un service des urgences est susceptible d'avoir subi des blessures sportives passées sous silence. La sous-estimation de ce taux de 6,7 \% pourrait atteindre 4,3\%. D’après les données de surveillance du SCHIRPT correspondantes, le taux d'incidence de ces blessures est de 9,9\%. Nous mettons également en lumière dans notre analyse le biais potentiel de masquage lié aux blessures traitées par d'autres prestataires de soins de santé.

Conclusion : Poser une question sur « la blessure la plus grave » risque d'induire un biais de masquage considérable du taux d'incidence des blessures sportives, limitant ainsi la capacité des chercheurs à estimer l'ampleur réelle du phénomène. Des périodes de rappel plus longues entraînent inévitablement un phénomène de masquage plus important. La conception des enquêtes à venir devrait tenir compte de ces réalités. Si l'on veut faciliter une prise de décisions éclairées et orienter correctement les futures recherches, il faut que les chercheurs soient conscients de ces limitations.

Mots-clés : jeunes, biostatistiques, épidémiologie, blessure la plus grave, blessure sportive, surveillance, enquête

\section{Points saillants}

- Les enquêtes recueillent souvent des données ne portant que sur la blessure la plus grave survenue durant une période de rappel particulière, afin de réduire le biais de rappel et de raccourcir la durée du sondage. Cependant, cette façon d'enquêter risque de masquer des traumatismes moins graves, entraînant une sous-estimation du taux d'incidence de certaines sous-catégories de blessures, par exemple celles survenant durant la pratique d'une activité physique ou d'un sport.

- Dans cette étude, nous avons utilisé des données tirées de l'Enquête sur les comportements liés à la santé des enfants d'âge scolaire (ECSEAS) de 2002 et du Système canadien hospitalier d'information et de recherche en prévention des traumatismes (SCHIRPT) pour comparer les estimations des taux d'incidence des blessures sportives chez les enfants canadiens. Des éléments de preuve d'un biais de masquage ont été observés.

- Sans une estimation exacte des taux d'incidence des sous-catégories de blessures, notre capacité à quantifier l'importance des traumatismes ainsi que la portée des interventions se trouve restreinte.

- On doit tenir compte de ce risque de masquage au moment de la phase de conception d'une enquête ainsi que lors de l'analyse et de l'interprétation des données. 


\section{Introduction}

Les blessures accidentelles entrainnent de graves problèmes de santé et sont considérées comme l'une des principales causes de mortalité chez les jeunes à l'échelle de la planète ${ }^{1,2}$. Une proportion importante de blessures chez les jeunes survient lors de la pratique d'un sport ou d'une activité physique (" blessures sportives $»)^{3,4,5}$. Des données non biaisées sur les blessures sportives sont donc susceptibles de contribuer à orienter les ressources en santé publique et à évaluer l'efficacité des efforts de prévention. À l'inverse, des estimations inexactes du taux de prévalence d'une sous-catégorie particulière de blessures rendent impossible une mise en lumière des bienfaits pour la santé des programmes de promotion de l'activité physique en regard des risques accrus de blessures sportives.

Diverses enquêtes de mesure de la santé, telles que l'Enquête sur les comportements liés à la santé des enfants d’âge scolaire (ECSEAS) ${ }^{2}$, l'Enquête mondiale réalisée en milieu scolaire sur la santé des élèves ("GSHS ») $)^{6}$, l'Enquête sur la santé dans les collectivités canadiennes (ESCC), l'Enquête longitudinale nationale sur les enfants et les jeunes (ELNEJ) ${ }^{7}$ et l'Enquête nationale sur la santé de la population (ENSP), utilisent des questions portant sur les circonstances entourant " la blessure la plus grave » subie par les répondants ${ }^{8}$. Il s'agit en général d'une blessure qui impose des limites à l'accomplissement des activités quotidiennes ${ }^{5,8,9}$ ou nécessite un traitement administré par un médecin ou une infirmière ${ }^{2,6,8}$. Cependant, parmi les enquêtes que nous avons étudiées, seule l'ECSEAS définit explicitement ce qui est entendu par " la blessure la plus grave ", à savoir la blessure qui " a mis le plus de temps à guérir $»^{2}$. Dans l'enquête GSHS, la question est la suivante : " $\mathrm{Au}$ cours des 12 derniers mois, qu'elle[sic] a été la blessure la plus grave que tu aies eu[sic]? » (entrainnant une réponse du type " J'ai eu un os fracturé ou une articulation démise $»)^{8}$. L'ESCC demande aux répondants ayant subi de multiples traumatismes de considérer « la blessure la plus grave » et de répondre à des questions contextuelles portant sur le mois, l'année, sa nature et le lieu où elle a été subie ${ }^{9}$. De même, l'ELNEJ demande : "Quelle a été la nature de la blessure la plus grave qu'il a subie? " (on utilise la déclaration des parents) avant d'interroger sur la nature et le lieu où cette blessure la plus grave a été subie ${ }^{10}$.

Le biais de rappel constitue une menace considérable à la validité des données recueillies dans le cadre d'une enquête sur les blessures. Des erreurs de rappel peuvent en particulier survenir lorsque la réponse d'un répondant est incomplète à cause d'une perte mémorielle (consécutive au passage du temps). L'exactitude des estimations reposant sur des enquêtes utilisant des périodes de rappel longues est donc davantage touchée par les limitations de la mémoire et du rappel ${ }^{11}$. Comme la perte mémorielle est plus faible lorsque la gravité des blessures est plus élevée ${ }^{12-13}$, la question portant sur « la blessure la plus grave " est susceptible de contribuer à réduire les biais de rappel, d'autant plus que le répondant n'a à rechercher dans ses souvenirs et à faire état des variables contextuelles qu'à propos d'un seul traumatisme, ce qui est susceptible à la fois de réduire de beaucoup le temps nécessaire pour remplir le questionnaire et d'améliorer l'exactitude et l'exhaustivité des réponses.

La collecte de données approfondies portant uniquement sur la blessure la plus grave présente néanmoins un défaut majeur : si un répondant signale par exemple que la blessure la plus grave subie au cours des 12 derniers mois est liée à un combat, toute autre blessure sportive, "moins grave ", subie durant la même période risque d'être passée sous silence. Cela entraine une sous-estimation du taux d'incidence des différentes catégories de blessures chez tout répondant ayant subi plus d'une blessure durant la période de rappel. Ce fait est souvent négligé, et l'ampleur de ce biais dans les estimations des taux d'incidence des blessures sportives n'a encore jamais été étudiée. Les estimations publiées, par exemple celle selon laquelle $66 \%$ des blessures chez les jeunes sont liées au sport $^{5}$, sont biaisées parce qu'elles ne s'intéressent qu'aux "blessures les plus graves ». Dans leur introduction, Billette et Janz $z^{5}$ précisent que leurs données ne rendent pas compte de toutes les blessures, mais ils ne font pas état de ce biais de masquage dans leur résumé (" points saillants $»)^{5}$. Tozija et collab. ${ }^{14}$ se sont appuyés sur les données de l'enquête GSHS pour affirmer que 29,3 \% des blessures subies par les adolescents sont survenues à l'école et Jildeh et collab. ${ }^{15}$ ont utilisé les données de l'ECSEAS pour affirmer que $78,5 \%$ des blessures se produisent à la maison, mais aucune de ces deux études n'a fait état du risque potentiel de biais de masquage lié à la collecte de données ne portant que sur « la blessure la plus grave».

\section{Méthodologie}

Pour explorer l'ampleur possible du masquage et du biais découlant d'une méthode de sondage portant exclusivement sur " la blessure la plus grave », nous avons estimé les taux d'incidence des blessures sportives chez les enfants canadiens à l'aide du cycle de 2002 des données tirées de l'ECSEAS et des données de surveillance de 2001 et de 2002 tirées du Système canadien hospitalier d'information et de recherche en prévention des traumatismes (SCHIRPT). Nous avons choisi cette période parce que les données actuelles de l'ECSEAS et du SCHIRPT ne sont pas directement comparables. À l'instar de Pickett et coll. ${ }^{16}$, nous avons utilisé les taux de deux hôpitaux affiliés au SCHIRPT du comté de Frontenac (Ontario). Ces sites ont constitué par le passé des indicateurs assez représentatifs des tendances nationales en matière de surveillance et, pris ensemble, ce sont les seuls sites du SCHIRPT à offrir la couverture intégrale d'une collectivité ${ }^{16}$.

Le SCHIRPT saisit les données concernant les blessures de toutes les personnes s'étant présentées avec une blessure au service des urgences de l'un des hôpitaux 
participants, alors que l'ECSEAS tient compte des blessures " les plus graves" traitées par un médecin ou une infirmière, que ce soit au cabinet d'un médecin de famille, en clinique, à l'hôpital, dans un dispensaire scolaire ou ailleurs, avec, depuis 2002, l'indication d'un traitement ou non dans un service des urgences (pour un aperçu des caractéristiques des populations ayant signalé une blessure sportive pour ces deux bases de données, voir tableau 1). Les données tirées de l'ECSEAS peuvent donc être utilisées pour estimer les taux d'incidence de toutes les blessures sportives traitées chez des enfants canadiens, à l'aide du pourcentage de répondants à l'ECSEAS affirmant avoir subi une blessure sportive, et elles peuvent aussi servir à estimer le taux d'incidence des blessures sportives traitées en service des urgences. Le taux de blessures sportives traitées en service des urgences devrait être plus directement comparable aux estimations du taux d'incidence fondées sur les données de surveillance, à savoir le nombre de blessures sportives pédiatriques recensées en service des urgences pour 100 enfants au sein de la population à risque. D'après le SCHIRPT, une blessure sportive est tout traumatisme subi durant la pratique d'un sport (sport organisé, exercice, leçons et entraînement), d'une activité récréative (luge, trampoline, jeannettes, scouts), de la danse à la maison, d'un jeu ou d'une escalade.

Les données de l'ECSEAS sont fondées sur la question de «la blessure la plus grave », qui (comme nous l'avons mentionné plus haut) risque de conduire à une déclaration incomplète des blessures sportives, ce qui fait qu'une estimation fondée sur un sondage " naïf » constitue en réalité la limite inférieure de l'étendue des estimations plausibles des taux d'incidence des blessures sportives. En utilisant d'autres données de l'ECSEAS afin d'établir si les répondants ont subi de multiples blessures durant la période de rappel, nous pouvons évaluer l'ampleur de l'éventuel masquage de blessures moins graves, dont les blessures sportives moins graves. Nous pouvons ainsi, grâce à elles, déterminer la limite supérieure de l'estimation des taux d'incidence des blessures sportives. Ce calcul correspond au pourcentage de répondants à l'ECSEAS soit ayant signalé une blessure sportive comme leur blessure la plus grave, soit ayant déclaré de multiples blessures et de ce fait pouvant avoir passé sous silence une blessure sportive " moins grave».

On a limité l'ensemble de données du SCHIRPT aux jeunes âgés de 11 à 15 ans au moment de la consultation au service des urgences, afin de le faire coïncider avec le groupe d'âge cible de l'ECSEAS. Le tableau 2 fournit une comparaison des données sur les blessures en fonction de ces deux sources de données.

\section{Résultats}

Parmi les 6688 personnes ayant répondu à l'ECSEAS en 2001-2002, 1766 (26,4\%) ont déclaré avoir subi leur blessure la plus grave au cours d'un jeu, d'un entraînement ou de la pratique d'une activité récréative (tableau 2). Cependant, 1994 enfants ont fait état de blessures multiples et seulement 1047 parmi eux ont signalé une blessure sportive comme étant la plus grave. Puisqu'il est possible que les 947 autres enfants aient subi des blessures sportives moins graves, ce sont donc 14,2\% des enfants qui peuvent avoir passé sous silence une blessure sportive, « masquée » à cause de la question centrée sur « la blessure la plus grave ». Les véritables estimations des taux d'incidence des blessures sportives tirées de l'enquête ECSEAS varient donc entre 26,4 et 40,6 enfants blessés pour 100 enfants par année, selon l'ampleur du masquage (tableau 3).

En sélectionnant les données liées aux blessures ayant nécessité un traitement en service des urgences, nous pouvons également obtenir des estimations des

TABLEAU 1

Données démographiques des personnes ayant signalé une blessure sportive pour chaque source de données

\begin{tabular}{|c|c|c|c|c|c|c|}
\hline & \multicolumn{2}{|c|}{$\begin{array}{c}\text { ECSEAS } \\
(\mathrm{n}=1766)\end{array}$} & \multicolumn{2}{|c|}{$\begin{array}{l}\text { ECSEAS SU } \\
(n=445)\end{array}$} & \multicolumn{2}{|c|}{$\begin{array}{l}\text { SCHIRPT } \\
(\mathbf{n}=\mathbf{8 8 4})\end{array}$} \\
\hline & $\begin{array}{c}\text { Nombre } \\
\text { (n) }\end{array}$ & $\begin{array}{c}\text { Pourcentage } \\
(\%)\end{array}$ & $\begin{array}{c}\text { Nombre } \\
\text { (n) }\end{array}$ & $\begin{array}{l}\text { Pourcentage } \\
(\%)^{\mathrm{b}}\end{array}$ & $\begin{array}{c}\text { Nombre } \\
\text { (n) }\end{array}$ & $\begin{array}{l}\text { Pourcentage } \\
(\%)\end{array}$ \\
\hline \multicolumn{7}{|l|}{ Sexe } \\
\hline Garçons & 861 & 48,8 & 223 & 50,1 & 562 & 63,6 \\
\hline \multicolumn{7}{|l|}{ Âge (ans) } \\
\hline 12 & 409 & 23,2 & 112 & 25,2 & 172 & 19,5 \\
\hline 13 & 384 & 21,7 & 105 & 23,6 & 203 & 23,0 \\
\hline 14 & 379 & 21,5 & 83 & 18,7 & 186 & 21,0 \\
\hline 15 & 357 & 20,2 & 97 & 21,8 & 171 & 19,3 \\
\hline
\end{tabular}

Abréviations : ECSEAS, Enquête sur les comportements liés à la santé des enfants d'âge scolaire; SCHIRPT, Système canadien hospitalier d'information et de recherche en prévention des traumatismes; SU, service des urgences.

${ }^{a}$ ECSEAS SU fait référence aux blessures pour lesquelles le répondant a demandé un traitement à un service des urgences.

${ }^{\text {b }}$ Pourcentage d'individus ayant demandé un traitement à un service des urgences. 
TABLEAU 2

Comparaison des données sur les blessures en fonction des trois sources de données

\begin{tabular}{|c|c|c|c|c|c|c|}
\hline \multirow[t]{2}{*}{ Rapport de blessures } & \multicolumn{2}{|c|}{$\begin{array}{l}\text { ECSEAS } \\
(n=6688)\end{array}$} & \multicolumn{2}{|c|}{$\begin{array}{l}\text { ECSEAS SU } \\
(n=6688)\end{array}$} & \multicolumn{2}{|c|}{$\begin{array}{l}\text { SCHIRPT } \\
(n=8896)\end{array}$} \\
\hline & Nombre & $\%$ & Nombre & $\%$ b & Nombre & $\%$ \\
\hline Nombre de blessés durant la période de rappel de 12 mois & 3652 & 54,6 & 896 & 13,4 & 1453 & 16,3 \\
\hline Blessure sportive $^{c}$ & 1766 & 26,4 & 445 & 6,7 & 884 & 9,9 \\
\hline Blessures multiples $^{d}$ & 1994 & 29,8 & 559 & 8,4 & - & - \\
\hline $\begin{array}{l}\text { Blessures multiples dans lesquelles la blessure la plus grave n'était } \\
\text { pas une blessure sportive }\end{array}$ & 947 & 14,2 & 287 & 4,3 & - & - \\
\hline
\end{tabular}

Abréviations : ECSEAS, Enquête sur les comportements liés à la santé des enfants d'âge scolaire; SCHIRPT, Système canadien hospitalier d'information et de recherche en prévention des traumatismes; SU, service des urgences.

${ }^{a}$ ECSEAS SU fait référence aux blessures pour lesquelles le répondant a demandé un traitement à un service des urgences.

${ }^{\text {b }}$ Proportion de répondants sur l'ensemble des répondants à l'ECSEAS (pas seulement par rapport à ceux ayant demandé un traitement à un service des urgences).

c Blessures les plus graves dans l'enquête ECSEAS et motifs de la consultation pour la surveillance des données SCHIRPT.

d II n'existe pas d'équivalent signalant les blessures les plus graves dans les données de surveillance.

taux d'incidence liés aux données d'enquête et de surveillance, et nous faire ainsi une meilleure idée de l'ampleur réelle du masquage. Durant la période de rappel de 12 mois, 896 enfants $(13,4 \%$ de la cohorte de l'ECSEAS) ont subi des blessures ayant requis un traitement à un service des urgences, et 445 de ces enfants ont mentionné une blessure sportive comme étant leur blessure la plus grave $(6,7 \%$ du total) (tableau 2). Parmi les 451 enfants ayant indiqué une blessure d'une autre sous-catégorie comme étant leur blessure la plus grave, 287 (4,3\% du total) ont indiqué avoir subi de multiples blessures. Ainsi, 4,3\% des enfants ont pu passer sous silence des blessures sportives (ce taux de masquage pourrait même être plus élevé en théorie si un enfant a jugé qu'une blessure n'ayant pas nécessité de traitement à un service des urgences était " plus grave » qu'une blessure l'ayant exigé). Les véritables estimations du taux d’incidence des blessures sportives nécessitant une consultation en service des urgences se situent donc entre 6,7 et 11,0 enfants (voire plus) pour 100 enfants et par année (tableau 3).

Les blessures sportives ont entraîné 884 consultations en service des urgences chez les 8896 enfants vivant dans la zone couverte par le SCHIRPT, soit environ $60,8 \%(n=884 / 1453)$ de toutes les blessures déclarées (tableau 2). Notre estimation du taux d'incidence des blessures sportives fondée sur les données de surveillance s'élève donc à 9,9 enfants pour 100 enfants et par année, valeur supérieure à l'estimation reposant sur un sondage naîf de 6,8, mais située à l'intérieur de la fourchette obtenue lorsque nous tenons compte du phénomène de masquage potentiel découlant de la question portant essentiellement sur « la blessure la plus grave " (tableau 3). En réalité, cette estimation fondée sur les données de surveillance plus élevée peut néanmoins constituer une sous-estimation du taux d'incidence réel, car les blessures sportives ne sont observées que chez les enfants dont les adultes responsables ont consenti à la collecte de données détaillées.

\section{Analyse}

L'objectif de cet article est d'illustrer l'existence d'un biais de masquage dans les enquêtes portant seulement sur «la blessure la plus grave ». L'impact potentiel d'une question axée sur « la blessure la plus grave » est évident sur nos

TABLEAU 3

Estimations des taux d'incidence des blessures sportives

\begin{tabular}{lc} 
& $\begin{array}{c}\text { Taux d'incidence des blessures sportives } \\
\text { (pour 100 enfants et par année) }\end{array}$ \\
\hline $\begin{array}{l}\text { Toutes blessures exigeant un traitement médical } \\
\text { ECSEAS }\end{array}$ & 26,4 à 40,6 \\
\hline $\begin{array}{l}\text { Blessures grraves exigeant un traitement médical à un SU } \\
\text { ECSEAS }\end{array}$ & 6,7 à 11,0 \\
SCHIRPT & 9,9 \\
\hline
\end{tabular}

Abréviations : ECSEAS, Enquête sur les comportements liés à la santé des enfants d'âge scolaire; SCHIRPT, Système canadien hospitalier d'information et de recherche en prévention des traumatismes. 
estimations des taux d'incidence des blessures sportives. En particulier, l'estimation liée au SCHIRPT $(9,9$ blessures sportives pour 100 enfants et par année) est proche des valeurs supérieures liées aux données de l'ECSEAS pour les services des urgences (de 6,7 à 11,0 blessures sportives pour 100 enfants et par année). Nous sommes ainsi amenés à penser que le masquage est un phénomène préoccupant. Le recours à cette question dans les enquêtes est certes susceptible d'entraîner une réduction du biais de rappel, mais les taux d'incidence des sous-catégories de blessures se trouveront alors sous-estimés $\mathrm{du}$ fait du masquage des blessures moins graves et les estimations de régression et celles du pourcentage de blessures entrant dans une sous-catégorie donnée sont susceptibles d'être biaisées, dans un sens ou dans l'autre, à cause de ce masquage. L'ampleur du masquage et du biais dépend du taux d'incidence, de la gravité relative et de la probabilité de survenue concomitante de sous-types de blessures spécifiques ainsi que de la probabilité de survenue de blessures multiples durant la période de rappel.

Ces facteurs indiquent que les données liées à la question portant sur " la plus grave blessure » ne sont pas appropriées lorsqu'on compare des estimations de taux d'incidence entre sous-groupes (p. ex. garçons par rapport à filles) susceptibles d'être touchés par ce masquage dans des proportions différentes. Les chercheurs doivent tenir compte des conséquences possibles de ce biais de masquage dans la conception et dans l'analyse des enquêtes sur les blessures. La question de savoir si d'autres blessures " moins graves » ont été enregistrées durant la période de rappel pourrait par exemple servir à obtenir un éventail d'estimations des taux d'incidence des blessures. Des périodes de rappel plus brèves permettraient quant à elles de saisir moins d'individus ayant subi de multiples blessures, ce qui amènerait une réduction du masquage d'événements et $\mathrm{du}$ biais. La collecte d'information sur toutes les blessures permettrait d'éliminer tout masquage. Cet avantage doit cependant être évalué à la lumière d'éventuels inconvénients associés à de telles modifications de la conception de l'étude, entre autres la perte de puissance et l'augmentation du biais de rappel.

Dans cet article, nous ciblons les circonstances entourant les blessures et nous faisons la preuve d'un biais, clair mais souvent négligé, affectant l'estimation des taux d'incidence lorsque sont utilisées les données axées sur " la blessure la plus grave ». Un biais du même ordre apparaît lorsqu'il s'agit d'estimer le taux d'incidence des effets des blessures (commotions, fractures, etc.), mais comparer les données d'enquête et les données de surveillance serait plus difficile parce que les répondants à l'ECSEAS peuvent déclarer jusqu'à cinq effets des blessures tandis que les données de surveillance du SCHIRPT n'en saisissent tout au plus que trois. Notons que le sens du biais des estimations des taux d'incidence est connu : le biais de masquage entraîne une sousestimation du taux d'incidence des sousgroupes de blessures. Les études d'association ou de taux relatifs pourraient cependant être biaisées dans un sens ou dans l'autre si l'ampleur du masquage différait entre groupes.

\section{Limitations}

Les données utilisées pour nos analyses remontent à 2001-2002, époque où des données d'enquête et des données de surveillance comparables étaient aisément disponibles. Les estimations des taux d'incidence des blessures sportives ne sont donc pas à jour.

Si la surveillance du SCHIRPT saisit toutes les personnes se présentant à un service des urgences avec une blessure, les renseignements détaillés, en particulier le contexte de la blessure, ne sont enregistrés que pour les enfants dont les parents ont consenti à participer à l'étude (les taux globaux de consentement étaient de $85 \%$ pour le site 1 et de $60 \%$ pour le site 2).

Même si la tranche d'âge ciblée par l'ECSEAS était les élèves de 11 à 15 ans en fonction de leur niveau scolaire, certains jeunes de 9 ans et de 19 ans ont aussi été intégrés à l'étude. Pour améliorer la comparabilité, nous avons limité nos ensembles de données aux sujets âgés de 11 à 15 ans pour le SCHIRPT comme pour l'ECSEAS.

La surveillance du SCHIRPT couvrant seulement la collectivité du comté de Frontenac, la comparaison des résultats de ce programme avec ceux de l'ECSEAS, qui est menée à l'échelle nationale, pose problème. Les données de population du SCHIRPT (comté Frontenac) et de l'ECSEAS diffèrent à certains égards. En particulier, les données du SCHIRPT ont porté sur un pourcentage plus élevé de garçons $(63,6 \%$, contre $48,8 \%$ dans l'ECSEAS) et la ventilation entre groupes d'âge s'est révélée un peu différente de celle de l'ECSEAS (p. ex. 17,2 \% d'enfants de 11 ans dans le SCHIRPT contre 13,4\% dans l'ECSEAS). Comme nous savons que les blessures varient en fonction de l'âge et du sexe, ces divergences risquent de se répercuter sur les résultats. Cependant, elles ne sont pas marquées au point d'ébranler sur notre constatation générale, qui est que les estimations des taux d'incidence des blessures sportives sont probablement biaisées et constituent une sous-estimation des taux d'incidence réels si elles reposent sur des données tirées d'une enquête posant la question de «la blessure la plus grave ».

\section{Conclusion}

Pour élaborer des programmes d'intervention et des politiques publiques axés sur la prévention des comportements à risque et pour contrer les conséquences de ces comportements chez les jeunes, les chercheurs et les décideurs doivent pouvoir compter sur des estimations fiables des taux d'incidence des blessures. Comme nos résultats le suggèrent, la différence entre les estimations des taux d'incidence des blessures du SCHIRPT et celles de l'ECSEAS est d'importance si nous ne tenons pas compte du biais de masquage altérant les données des enquêtes. Si la question de " la blessure la plus grave " permet de surmonter certains des biais traditionnels associés aux données d'enquêtes, les estimations des blessures sportives (ou de toute autre sous-catégorie de blessures) ne devraient être utilisées que si on fait état des biais potentiels et du 
masquage inhérents. Les taux d'incidence de blessures sportives spécifiques sont sous-estimés par les analyses naïves d'enquêtes ne collectant des données que sur « la blessure la plus grave ». Ces analyses devraient plutôt faire état de fourchettes d'estimations des taux d'incidence plausibles qui rendent compte des éventuels niveaux de masquage. Ainsi, dans la conception des études à venir, il y a lieu de tenir compte du masquage provenant de la question axée sur " la blessure la plus grave " ainsi que de divers problèmes comme le biais de rappel. Nous encourageons également fortement la collecte de données permettant de découvrir si les répondants à l'enquête ont subi d'autres blessures " moins graves ", afin de faciliter l'exploration de l'ampleur d'un potentiel biais de masquage.

\section{Conflits d'intérêts}

Les auteurs n'ont aucun conflit d'intérêts à déclarer.

\section{Remerciements}

Akshay Gupta a bénéficié du soutien à titre de stagiaire de recherche pour la période estivale de la part de la Child and Youth Injury Prevention Team (équipe de prévention des blessures chez les enfants et chez les jeunes (http://www .childinjuryprevention.ca) des IRSC et il a reçu l'appui de la Section des blessures, Division de surveillance de la santé et de l'épidémiologie de l’Agence de la santé publique du Canada (contrat 4500307663).

Les auteurs tiennent à remercier Wendy Thompson et Mylène Fréchette de l'Agence de la santé publique du Canada ainsi que le docteur Robert Brison du Département de médecine d'urgence de l'Hôpital général de Kingston pour leur aide dans la collecte et l'interprétation des données du SCHIRPT dont ils ont eu besoin ainsi que pour leurs avis et suggestions. Nous aimerions aussi remercier Don McGuiness, du réseau local d'intégration des services de santé du Sud-Est, pour nous avoir fourni les données de la population du secteur.

L'ECSEAS et le SCHIRPT sont financés par l’Agence de la santé publique du Canada (http://www.phac-aspc.gc.ca/index-fra.php) et par Santé Canada (http://www.hc-sc.gc.ca /index-fra.php). L'ECSEAS est le fruit d'une collaboration avec l'Organisation mondiale de la santé et, en 2002, elle était coordonnée par la docteure Candace Currie (Université de St. Andrews, Écosse). Le volet canadien de l'enquête est coordonné par Mathew King et dirigé par les docteurs John Freeman et William Pickett (Université Queen's, Canada).

\section{Références}

1. Currie C, Gabhainn SN, Godeau E et collab. (dir.). Inégalités en matière de santé chez les jeunes. Rapport international HBSC issu de l'étude 2005/2006. Genève : Organisation mondiale de la santé; 2008. Résumé en français (et lien vers le téléchargement du rapport complet en anglais) à la page http://www.euro.who. int/fr/health-topics/Life-stages/child-and -adolescent-health/publications/2008 / inequalities-in-young-peoples -health.-hbsc-international-report-from -the-20052006-survey

2. Pickett $\mathrm{W}$, Molcho $\mathrm{M}$, Simpson $\mathrm{K}$ et collab. Cross national study of injury and social determinants in adolescents. Inj Prev. 2005;11:213-218.

3. Scheidt PC, Harel Y, Trumble AC, Jones $\mathrm{DH}$, Overpeck MD, Bijur PE. The epidemiology of nonfatal injuries among US children and youth. Am J Public Health. 1995;85(7):932-938.

4. Pakzad-Vaezi K, Singhal A. Trends in paediatric sport- and recreation-related injuries: An injury surveillance study at the British Columbia Children's Hospital (Vancouver, British Columbia) from 1992 to 2005. Paediatr Child Health. 2011;16(4):217-221.

5. Billette JM, Janz T. Les blessures au Canada : Un aperçu des résultats de l'Enquête sur la santé dans les collectivités canadiennes. 2011. Coup d'œil sur la santé, juin 2011. Ottawa (Ont) : Statistique Canada; 2011. [Statistique Canada, $\left.\mathrm{n}^{\circ} 82-624-\mathrm{X}\right]$.

6. Turagabeci AR, Nakamura K, Takano T. Healthy lifestyle behaviour decreasing risks of being bullied, violence and injury. PloS One. 2008;3(2):e1585. doi: 10.1371/ journal.pone.0001585.
7. White HL, Macpherson AK. Capturing paediatric injury in Ontario: differences in injury incidence using self-reported survey and health service utilisation data. Inj Prev. 2012;18(1):33-7. doi: 10.1136/ injuryprev-2011-040006.

8. Centers for Disease Control and Prevention. Global School-Based Student Health Survey (GSHS) 2013 Core Questionnaire Modules: Final. Atlanta (GA): Centers for Disease Control and Prevention; 2013.

9. Statistique Canada. Enquête sur la santé dans les collectivités canadiennes (ESCC) : composante annuelle - Questionnaire de 2014. Statistique Canada; 2014.

10. Statistique Canada. Enquête longitudinale nationale sur les enfants et les jeunes : matériel d'enquête pour la collecte 20082009, Cycle 8. Statistique Canada; 2008.

11. Warner M, Schenker N, Heinen MA, Fingerhut LA. The effects of recall on reporting injury and poisoning episodes in the National Health Interview Survey. Inj Prev. 2005;11:282-7.

12. Moshiro C, Heuch I, Astrom AN, Setel P, Kvale G. Effect of recall on estimation of non-fatal injury rates: a community based study in Tanzania. Inj Prev. 2005;11:48-52.

13. Coughlin SS. Recall bias in epidemiologic studies. J Clin Epidemiol. 1990;43(1):87-91.

14. Tozija F, Dragan G, Kasapinov B, Nikovska DG. Youth injury risk behavior and safety in Macedonia. Inj Prev. 2010;16(Suppl 1);A83-4. doi:10.1136/ip.2010.029215.304.

15. Jildeh C, Abdeen $\mathrm{Z}, \mathrm{Al}$ Sabbah $\mathrm{H}$, Philalithis A. Unintentional injuries among school-aged children in Palestine: findings from the National Study of Palestinian Schoolchildren (HBSC-WBG2006). Int J Population Research. 2013;2013:1-7. doi. org/10.1155/2013/629159.

16. Pickett W, Brison RJ, Mackenzie SG et collab. Youth injury data in the Canadian Hospitals Injury Reporting and Prevention Program: do they represent the Canadian experience? Inj Prev. 2000;6:9-15. 